\title{
Serbische Salonmusik im Kontext der gesellschaftlichen Modernisierung in der ersten Hälfte des 19. Jahrhunderts
}

\section{Einleitung}

Anlässlich der wissenschaftlichen Auseinandersetzung mit der Salonmusik wie auch der serbischen Kultur des 19. Jahrhunderts insgesamt ist es unerlässlich, auch das Bestehen des kulturellen Pluralismus im Blick zu haben, der sich durch die komplexen und dynamischen sozial-historischen Bewegungen erklärt sowie durch die Tatsache, dass sich das private und öffentliche Leben des serbischen Volkes in unterschiedlichen sozialen und staatlichen Systemen abspielte: in der Habsburgermonarchie bzw. Österreichisch-Ungarischen Monarchie, dem Osmanischen Reich und dem Fürstentum bzw. Königreich Serbien. ${ }^{1}$ Seitdem die letzten serbischen Gebiete des mittelalterlichen Staates unter osmanische Herrschaft gefallen waren (1459) bis hin zum Beginn des 19. Jahrhunderts existierte Serbien als eigener Staat nicht. Zur Zeit der Türkenkriege vom 16. bis zum 18. Jahrhundert kam es zu großen Migrationsbewegungen der Serben Richtung Norden (in den Süden Ungarns) und nach Nordwesten (Kroatien), die größte Völkerwanderung fand 1690 unter der Führung des Patriarchen Arsenije III. Čarnojević statt. So wurde der römisch-deutsche Kaiser in Wien zum Souverän von rund 100.000 Serben in der kaiserlichen Militärgrenze zwischen der oberen Adria und den Südkarpaten sowie in jenen Regionen, die der zivilen Herrschaft der Habsburger unterstanden (Banat, Bačka und Srem). ${ }^{2}$ Der Befreiungskampf der Serben im Osmanischen Reich erhielt besonders zu Beginn des 19. Jahrhunderts einen neuen Aufschwung und entflammte im Ersten (1804) und Zweiten serbischen Aufstand (1815), woraufhin das Fürstentum Serbien entstand, welches 1882 zum Königreich ${ }^{3}$ ausgerufen wurde. Mit der Befreiung von der Osmanischen Herrschaft führten die Serben nicht nur eine nationale Revolution an, sondern auch eine soziale Revolution, wodurch entscheidende Veränderungen in der Gesellschaft ermöglicht wurden, nämlich hinsichtlich jenes Zeitgeistes, auf Grundlage dessen sich das damalige moderne Europa weiterentwickelte. ${ }^{4}$ Einen bedeutenden Einfluss auf die Bür-

1 Diese Studie ist das Resultat einer Arbeit am Projekt Identitäten der serbischen Musik von lokalen zu globalen Auswirkungen: Traditionen, Veränderungen, Herausforderungen (ON 177004), das vom Bildungs- und Unterrichtsministerium der Republik Serbien finanziert wurde.

2 Danica Petrović, „Serbien“, in: Oesterreichisches Musiklexikon Online, hrsg. von Rudolf Flotzinger, Wien 2002-2015, <http://www.musiklexikon.ac.at/ml/musik_S/Serbien.xml>, ISBN: 978-3-70013077-2, 15.04.2015.

3 Das Fürstentum Serbien wurde 1867 durch Fürst Mihailo Obrenović, den Sohn des Fürsten Miloš Obrenović, zur Gänze vom osmanischen Heer befreit. Dies geschah, indem er den Rückzug des osmanischen Herres aus den Garnisonen des Fürstentums Serbien erwirkte. Ein international anerkannter, unabhängiger Staat wurde das Fürstentum Serbien durch die Beschlüsse auf dem Berliner Kongress im Jahr 1878.

4 Leopold Ranke hat als Erster den Ausdruck „serbische Revolution“ in seinem gleichnamigen Buch (1829) benutzt, in welchem er auf das noch nicht erreichte Ziel hinwies: die Erschaffung eines Rechtsstaates und die Bildung des Volkes. Dadurch nämlich würde es „zur Teilnahme am geistigen Leben kommen, was zum wahren Glück führt“. Unter dem geistigen Leben verstand Ranke die „Predigt des 
gerlichkeit in Serbien hatte die serbische Bürger-Elite in der Habsburgermonarchie, die sich bereits im 18. Jahrhundert herauszubilden begann. Dank der wirtschaftlichen Kontakte, der Familienbindungen sowie der Migrationsbewegungen einer bedeutenden Anzahl gebildeter Bürger aus der Habsburgermonarchie im Fürstentum und später im Königreich Serbien wurden kulturelle Gebräuche und Ideale in das bürgerliche Privatleben übernommen. ${ }^{5}$

Die serbische Kunst und Kultur erfuhr im 18. und 19. Jahrhundert innerhalb der Habsburgermonarchie eine bedeutende Entwicklung. Zahlreiche Einflüsse, vor allem aus Mitteleuropa, aber auch aus Russland, fanden über das Gebiet des südlichen Ungarns Eingang in die serbische Kultur. Zur damaligen Zeit lebte die serbische Bevölkerung im Rahmen des Osmanischen Reiches unter völlig anderen gesellschaftlich-historischen Bedingungen. Angespornt durch die kulturellen Geschehnisse in der unmittelbaren Umgebung orienterte sich das serbische Bürgertum an der Lebensweise der österreichischen und ungarischen Aristokratie. Ende des 18. Jahrhunderts wurden die wichtigsten Schulen gegründet, welche im nachfolgenden Jahrhundert die gesamte musikalische Bildung der Serben in der Habsburgermonarchie übernahmen: die Präparandie (Vorbereitungsanstalt zur Lehrerausbildung) in Sombor (1778), das Gymnasium (1791) sowie das Priesterseminar (1794) in Sremski Karlovci und das große serbisch-orthodoxe Gymnasium in Novi Sad (1810). ${ }^{6}$ Der erste ausgebildete serbische Musiker, Pianist und Komponist Kornelije Stanković (1831-1865) genoss seine anfängliche Musikausbildung in Arad, Szeged und Budapest und setzte sie dann in Wien fort, wo er Musiktheorie und Komposition bei Simon Sechter lernte. Seine Vorgänger (Aleksandar Morfidis Nisis, Milan Milovuk, Nikola Đurković, Spiridon Trbojević) und Nachfolger (Aksentije Maksimović, Mita Topalović, Jovan Paču, Isidor Bajić) bereicherten das Musikleben auf vielfältige Weise: als Komponisten, als Dirigenten von Gesangsvereinigungen und Kirchenchören sowie als Pädagogen und Sammler von volkstümlichen Melodien. Mangels einheimischer Musikexperten und unter dem Einfluss panslavischer Ideen wurden tschechische Musiker ins Land berufen, welche in der zweiten Hälfte des 19. Jahrhunderts eifrig an der Weiterentwicklung des Musiklebens - insbesondere in den Provinzen - mitgewirkt haben (Gvido Havlas, Václav Horejšek, Hugo Doubek, Robert Tollinger u. a.). Einen bedeutenden Beitrag für die rasche Entwicklung des Musiklebens leisteten auch hervorragende ausführende Musiker wie etwa die Pianistin Jovanka Stojković

Christentums in seiner Reinheit und die Weitergabe der europäischen wissenschaftlichen Errungenschaften" in Serbien. Leopold Ranke, Srpska revolucija [dt: Die serbische Revolution], Übersetzung auf serbisch: Ognjan Radović, Belgrad 1991, S. 164.

5 Darko Ivanović, Srpski učitelji iz Habzburške monarhije u Srbiji (1804-1858) [dt: Serbische Lehrkräfte aus der Habsburgermonarchie in Serbien], Belgrad 2006, S. 5-8.

6 Danica Petrović, „Srpsko pojanje i Karlovačka mitropolija u XVIII veku“ [dt.: Serbischer Gesang im Metropolitansitz Sremski Karlovci im 18. Jahrhundert], in: Pro musica 148 (1992), S. 5-8. 
(1855-1892), ${ }^{7}$ der Violinist Dragomir Kranjčević (1847-1929), ${ }^{8}$ aber auch insbesondere zahlreiche Kirchenchöre und Gesangsvereinigungen, die unmittelbar nach der Gründung der ersten serbischen Gesangsvereinigung in Pančevo (1838) auch in anderen serbischen Städten entstanden sind.

Bei den Serben im Osmanischen Reich begann das kulturelle Leben nach dem zweiten serbischen Aufstand, während der ersten Herrschaft des Fürsten Miloš Obrenović (17831860). ${ }^{9}$ In das junge Fürstentum Serbien kamen nach dem Jahr 1830 aus der Habsburgermonarchie Josif Schlesinger (1794-1870), Alois Kalauz ${ }^{10}$ und Milan Milovuk (18251883), welche einen bedeutenden Beitrag bei der Gründung der ersten Musikinstitutionen und bei der Verbreitung musikalischer Bildung geleistet haben. Schlesinger gründete das erste Orchester in Serbien (1831) und komponierte Musik für das Fürstlich-serbische Theater für das damals beliebte Genre „Theaterstücke mit Gesang“ (eine Art des heimischen Singspiels). So wurde das deutsche Singspiel, welches sich bei den Serben in Ungarn großer Beliebtheit erfreute, auch in Serbien mit Begeisterung aufgenommen. Die erste Belgrader Gesangsvereinigung wurde im Jahre 1853 von Milan Milovuk gegründet, ${ }^{11}$ der aus Budapest nach Belgrad gekommen war. Sein Wirken in der Vereinigung setzte K. Stanković nach seiner Rückkehr aus Wien fort. Im Rahmen dieser Gesangsvereinigung wurde 1899 die erste Musikschule in Serbien eröffnet. Alle bedeutenderen serbischen Komponisten aus

7 Sie wurde 1852 in Temesvar geboren. Neben ihrer Karriere als Pianistin wirkte sie auch als Konzertsängerin, Komponistin und Musikpädagogin. Sie lebte bis zu ihrem 18. Lebensjahr in Prag und Wien. Klavierunterricht erhielt sie von Alexandar Dreyschock, dem hervorragenden tschechischen Pianisten, und später bei Franz Liszt. Ihre musikalische Ausbildung setzte sie in Mailand fort, wo sie zwei Jahre lang neben Klavier auch Gesang studierte. Sie trat in vielen Städten Serbiens und Österreich-Ungarns auf sowie in England, Frankreich, Dänemark und Russland. Ende 1879 ließ sie sich in Belgrad nieder, wo sie als Pianistin und Privatlehrerin tätig war. Das letzte Konzert gab sie in Serbien 1879, von da an gibt es über sie in der serbischen Presse keine Erwähnungen mehr. Sie starb 1892 in Paris. Marijana Kokanović Marković, „Zur Geschichte der Beethoven-Rezeption in Novi Sad und Belgrad im 19. Jahrhundert: Jovanka Stojković und Beethovens Klaviersonaten", in: Beethoven-Rezeption in Mittel- und Osteuropa, hrsg. von Helmut Loos, Leipzig 2015, S. 139-149.

8 Geboren ist er in Pančevo, und in Wien studierte er bei Josef Hellmesberger sen. Geige. Zeitgleich studierte er Musiktheorie, sowie Klavier. 1859 trat er vor Henri Vieuxtemps auf, der sich über das jugendliche Musizieren sehr lobend äußerte. In Wien lernte er Hans von Bülow, Clara Schumann, Johannes Brahms und Joseph Joachim kennen. Neben Konzerten in serbischen Städten trat er auch in Österreich und Deutschland auf. Von 1873 bis 1901 wirkte er als Konzertmeister an der Oper in Budapest. Mihovil Tomandl, „Dragomir Krančević“, in: Muzika 5 (1951), S. 1-23; Stana Đurić-Klajn, „,Dragomir Krančević“, in: Pro musica 20 (1966), S. 6-8.

9 Durch die Befreiung Serbiens von der türkischen Herrschaft wurden die Bedingungen für die Entwicklung der Städte geschaffen, die in jener Periode zu Zentren der Verwaltung, der Bildung, des Handels und der Kultur wurden. Es kommt auch zur Migration der serbischen Dorfbevölkerung in die Städte, in welche ebenso jene Flüchtlinge gelangen, die nach der Bedrängnis während des ersten serbischen Aufstands nach Österreich geflüchtet waren. Durch die Vereinigung der ankommenden Menschen mit der autochthonen Stadtbevölkerung (griechische, zinzarische, muslimische) kam es zur Durchmischung von traditionellen, orientalischen und europäischen Kulturelementen. Slobodan Zečević, Srpske narodne igre [Serbische Volkstänze], Belgrad 1983, S. 28.

10 Geburts- und Sterbejahr sind nicht bekannt.

11 Unter der Schirmherrschaft der Belgrader Gesangsvereinigung leitete Milovuk auch eine private Musikschule. Er veröffentlichte auch die ersten Theoriebücher über Musik in serbischer Sprache: Teorički osnovi muzike [dt.: Theoretische Grundlagen der Musik] (1866) und Nauka o muzici [dt.: Wissenschaft über die Musik] (1867). Zudem komponierte er internationale Salontänze für Klavier, die sich großer Beliebtheit erfreuten. 
der zweiten Hälfte des 19. Jahrhunderts wirkten in dieser Schule mit: neben K. Stanković und Davorin Jenko (1835-1914) ${ }^{12}$ auch Josif Marinković (1851-1931) und Stevan S. Mokranjac (1856-1914). Parallel zur Wandlung Belgrads von einer orientalischen in eine moderne europäische Stadt entwickelte sich dementsprechend auch das musikalische Leben, und zahlreiche musikalische Institutionen entstanden: Gesangsvereine, Kammerensembles, Theater mit Singspiel und letztlich mit bekannten Opern und Operetten im Programm, Musikschulen, Theater- und Militärorchester. Obwohl staatlich-politische Grenzen und unterschiedliche gesellschaftliche Lebensumstände in der ersten Hälfte des 19. Jahrhunderts eine Barriere zwischen den Serben in der Habsburgermonarchie und dem Fürstentum Serbien bildeten, verlief das Musikleben der südlichen Städte in der Vojvodina und Belgrads bereits seit den 1840er Jahren trotzdem parallel und ergänzte sich gegenseitig. Besonders offenkundig wurde dies in der Tätigkeit der frisch eröffneten Gesangsvereinigungen und Theater, aber auch im Wirken der serbischen und ausländischen (besonders tschechischen) Komponisten.

\section{Klavier und Musikausbildung als Zeichen der gesellschaftlichen Modernisierung}

Im Unterschied zu Regionen mit reicher musikalischer Tradition bedeutete das Aufkommen von Salonmusik bei den Serben nicht nur eine „Richtung“ in der Popularmusik jener Zeit, die parallel zur Kunstmusik existierte, sondern sie fällt zeitlich mit dem Erscheinen der ersten Komponisten und Musikausgaben zusammen, die vorwiegend genau der Domäne der Salonmusik angehörten. Somit wurde - im Unterschied zu entwickelteren Musikzentren - auf gewisse Weise gerade durch die Salonmusik auch die Kunstmusik „geboren“. Die Spuren von Salonmusik gehen bei den reicheren Serben in der Habsburgermonarchie bis in die letzten Jahrzehnte des 18. Jahrhunderts zurück. Im gedruckten Katalog der Buchhandlung Emanuel Janković in Novi Sad aus dem Jahr 1790 sind neben Büchern auch Notenausgaben aufgelistet, die dort käuflich erwerbbar waren, was ein deutlicher Hinweis auf das Bestehen von Spinetten, Klavieren und Streichinstrumenten in den Häusern der reicheren Bewohner Novi Sads gegen Ende des 18. Jahrhunderts ist. ${ }^{13}$ Serbische Schriftsteller haben in ihren Werken ebenso auf die Bedeutung des Klavierunterrichts bei der Jugend, insbesondere bei Mädchen, hingewiesen. Jovan Sterija Popović erwähnt in seinem 1830 in Vršac erschienenen Werk Pokondirena tikva [Der hochmütig gewordene Kürbis], dass das Fortepiano bereits „seit langem in Mode“ sei und dass es für ein Mädchen aus angesehener Familie eine Schande sei, wenn es keine Virtuosin auf dem Klavier wäre. ${ }^{14}$

12 Der Slowene Davorin Jenko kam 1862 als Chorleiter der serbischen Gesangsvereinigung nach Pančevo, um später Chorleiter der Belgrader Gesangsvereinigung (1865-1877) zu werden. Zuvor war er während seiner Studienzeit in Wien in Berührung mit panslawischen Ideen gekommen. Von 1871 an wirkte er als Kapellmeister am Serbischen Nationaltheater in Belgrad. Er ist einer der produktivsten Komponisten von Bühnenmusik in Serbien im untersuchten Zeitraum und zugleich Komponist der bis heute gültigen serbischen Nationalhymne „Bože pravde“ [dt.: Gott der Gerechtigkeit].

13 Josip Andreis, Dragotin Cvetko, Stana Đurić-Klajn, Historijski razvoj muzičke kulture u Jugoslaviji [dt.: Geschichtliche Entwicklung der Musikkulturen in Jugoslawien], Zagreb 1962, S. 570.

14 Jovan Sterija Popović, „Pokondirena tikva“ [dt.: Der hochmütig gewordene Kürbis], in: Komedije, Novi Sad, Belgrad 1970, S. 168. Stevan Sremac kommentierte sechzig Jahre später in seiner Komödie Pop Ćira i pop Spira [„Popen sind auch nur Menschen“, 1894] auf geistreiche Weise, dass die Haupttugenden der Mädchen „Klavier, Häckelei, Walzer und Deutsch“ wären. 
Das häusliche Musizieren stellte in den Häusern wohlhabender Bürgerfamilien eine bevorzugte Art der Unterhaltung dar. ${ }^{15}$ Das Verfügen über ein Klavier, über einen privaten Musiklehrer sowie über eine Hausbibliothek mit edierten Notenausgaben gab Auskunft über den gesellschaftlichen Status. So wurde zum Beispiel der allgemeine Unterricht sowie der Musikunterricht für Mädchen aus angesehenen Familien der Stadt Novi Sad vorzugsweise Aleksandar Morfidis Nisis (1803-1878) anvertraut. ${ }^{16}$ Seit 1838, als er von Wien nach Novi Sad zog, bis zu seinem Tod war Morfidis Nisis die treibende Kraft des Musiklebens der Stadt: als Musiklehrer im Serbischen Gymnasium, als Klavierlehrer für Kinder aus angesehenen Familien Novi Sads, als Chorleiter und Komponist sowie als Organisator des Musiklebens. Im Klavierunterricht wandte er die Methode von Friedrich Kalkbrenner an. Neben dem Klavierunterricht lehrte er den Schülern die deutsche und französische Sprache sowie Ästhetik nach dem Buch Briefe an eine Jungfrau über die Hauptgegenstände der Aesthetik von Christian Oesers. Abgesehen von Privatunterricht wurden Mädchen aus angesehenen Familien der Stadt Novi Sad auch in sogenannten Mädchenanstalten ausgebildet und erzogen (z. B. in den Anstalten von Helene Spieler, Anna Trenz, Anna Kranzl, Terezija Miković, Alojzija Heger). In den Lehrplänen der Mädchenanstalten, die im Untersuchungszeitraum in der Vojvodina existierten, lag der Schwerpunkt auf Fremdsprachen, Klavierspiel und Handarbeiten. Zu einer der bekannteren Anstalten in Novi Sad zählte jene, die von der gebürtigen Schweizerin Amelie Terzić geb. Moreau geleitet wurde. Im Lehrplan stand, dass das Erlernen der deutschen, der ungarischen, der serbischen und der französischen Sprache verpflichtend war. Zusätzlich lernten die Mädchen Klavier und Handarbeiten. ${ }^{17}$

Bemerkenswerterweise tauchen die ersten Klaviere in Serbien in den 1820er Jahren auf, und zwar für die Bedürfnisse der Fürstenfamilie Obrenović. Das erste Klavier wurde auf Ansuchen des Fürsten Miloš Obrenović geliefert und im Haus des fürstlichen Leibarztes, dem Italiener Vito Romita, untergebracht. Bei der Ehefrau des Arztes erwarb Prinzessin Jelisaveta (Savka) Obrenović neben Kenntnissen im Klavierspiel auch die „europäische Bildung“. Prinzessin Anka, die Tochter von Jevrem Obrenović, dem Bruder des2 Fürsten, lernte Klavier bei Josif Schlesinger, der zu dieser Zeit von Novi Sad nach Serbien zog. Besonders bedeutend ist aus musiksoziologischer Perspektive die Tatsache, dass ein Klavier „erworben wurde von Angehörigen reichster und mächtigster Familien in Serbien, so dass sie deren Töchtern - Vertretern der erst sich herausbildenden höfischen Elite - das Musizieren auf demselben ermöglichten und damit noch mehr den privilegierten Status hervorhoben ". ${ }^{18}$ Das Klavier fand sehr schnell Eingang in die höheren Bürgerschichten und wurde grundle-

15 Marijana Kokanović Marković, Društvena uloga salonske muzike u životu i sistemu vrednosti srpskog gradanstva u 19. veku [dt.: Die gesellschaftliche Rolle der Salonmusik im Leben und Wertesystem des serbischen Bürgertums im 19. Jahrhundert], Belgrad 2014, S. 116-141.

16 Über seine Herkunft gibt es keine zuverlässigen Daten. Seine Kindheit verbrachte er in Wien. Er studierte Medizin, schloss sein Studium aber nicht ab, da ihn die Musik mehr faszinierte. Er war ein begabter Sänger (Bass). Nach dem Tod seiner Mutter kam er mit Kaufleuten nach Novi Sad. Während der Revolution 1848 unterbrach er für kurze Zeit sein Wirken als Musikpädagoge und begab sich nach Osijek und Rijeka. Er lebte ein stürmisches Leben als Bohémien und starb im Krankenhaus an den Folgen eines Schlaganfalls. Marijana Kokanović Marković, „Aleksandar Morfidis Nisis“, in: Iz novosadskih salona - Album salonskih kompozicija za klavir [dt.: Aus den Salons Novi Sads - Album mit Salonwerken für Klavier], hrsg. Marijana Kokanović, Novi Sad 2010, S. 21.

17 Vasa Stajić, Grada za kulturnu istoriju Novog Sada [dt.: Bausteine zur Kulturgeschichte Novi Sad], Novi Sad 1951, S. 187-191.

18 Dragana Jeremić Molnar, Srpska klavirska muzika u doba romantizma [dt.: Serbische Klaviermusik im Zeitalter der Romantik], Novi Sad 2006, S. 35. 
gender Bestandteil in den Salons reicher Kaufleute, Beamter, Intellektueller sowie zugezogener Nichtserben. Ebendiese hatten einen großen Einfluss auf die Pflege der Hausmusik, insbesondere in den Provinzen. Ab 1843 wirkte in Belgrad der Tscheche Alois Kalauz, der als Pianist auftrat und privaten Klavierunterricht gab. In der zweiten Hälfte des 19. Jahrhunderts wird diese von ausländischen Musiklehrern begonnene Linie von einer Reihe tschechischer Musiker fortgesetzt.

Es ist offensichtlich, dass vorerst beinahe ausschließlich Fremde Klavierunterricht gaben, die die europäischen Kulturwerte in die neue Heimat mitbrachten. Neben primär pädagogischem Wirken widmeten sie sich auch dem Aufbau eines öffentlichen Musiklebens, indem sie Konzerte organisierten, auf welchen sie als Pianisten eigenständig oder gemeinsam mit ihren Schülern auftraten. Um den Wünschen der neuen Umgebung entgegenzukommen, komponierten sie Salonmusik für Klavier, in welchen sie oft Melodien serbischer Volkslieder und -tänze bearbeitet haben.

\section{Zwischen internationalen Tänzen und Bearbeitungen von Volksliedern: Komponisten und Genres von Salonmusik}

Seit der Zeit von Kornelije Stanković trugen Morfidis Nisis in Novi Sad Alois Kalauz in Belgrad sowie Josif Schlesinger in beiden Städten mit deren Tätigkeit als Pädagogen, Musiker und Komponisten nennenswert für die grundlegende Entstehung eines Musiklebens bei. Als paradigmatische Beispiele seien das Schaffen von Nisis und Kalauz angeführt, da Schlesingers Klavierwerke großteils Transkriptionen seiner Orchesterwerke sind. Schlesinger komponierte in erster Linie für das Orchester der Stadtgarde in Novi Sad, und später für die serbisch-fürstliche Musikkapelle in Kragujevac. Neben Märschen nehmen in seinem Schaffen auch gesellschaftliche Salontänze einen bedeutenden Platz ein, die er für Bälle und unterschiedliche Anlässe komponiert oder auch nur arrangiert hat. Franjo Kuhač weist darauf hin, dass Schlesinger Kontakte mit Wiener Musikkreisen hatte, vor allem mit Simon Sechter, mit dem Pianisten Joseph Fischhof (1804-1857) und mit Johann Strauss Vater (1804-1849) ${ }^{19}$. Auch Nisis und Kalauz sind Ankömmlinge aus Österreich, welches als Zentrum der panslawischen Elite auch in der nachfolgenden Periode eine wesentliche Rolle spielen sollte, wenn es um das Musikleben bei den Serben ging. In Novi Sad entwickelte Nisis 1838 sein vielfältiges Wirken und fünf Jahre später Kalauz in Belgrad. Im kompositorischen Schaffen beider Musiker dominieren internationale Salontänze für Klavier sowie Bearbeitungen von serbischen Volkstänzen und bürgerlichen Liedern und Tänzen, insbesondere Variationen für Klavier. Durch ihre Tätigkeit insgesamt wurden sie auch in der frühen serbischen Musikgeschichtsschreibung als Vorgänger von Kornelije Stanković angeführt.

Neben seinem vorrangig pädagogischen Wirken hat A. Morfidis Nisis für Auftritts- und Konzertanlässe, bei welchen er nicht selten auch selbst mit seinen Schülern auftrat, Salonmusik für Klavier komponiert, ebenso wie auch Kirchenkompositionen, welche vom Chor der Kirche des Hl. Georg in Novi Sad gesungen wurden. Und es ist sein Walzer Pozdrav srbskim djevama Op. 1 (1841, dt.: Gruß an die serbischen Jungfrauen), der in der serbischen Musikgeschichte als erstes, bis dato bekanntes gedrucktes Werk für Klavier gilt. ${ }^{20}$ In diesem

19 Franjo Kuhač, ,Josif Šlezinger. Prvi srpski kapelnik knjaževske garde“ [dt.: Josif Schlesinger. Erster serbischer Kapellmeister der fürstlichen Garde], in: Vienac: zabavi i pouci 8 (1897), S. 126.

20 Jeremić Molnar, Srpska klavirska muzika u doba romantizma [Serbische Klaviermusik im Zeitalter der Romantik], S. 8. 
Werk findet sich auch Nisis' Bewunderung gegenüber Robert Schumann bestätigt, was auch im Briefwechsel des Komponisten sowie in den Widmungen für seine Schüler offenkundig wird. Nisis hat den Walzer Gruß an die serbischen Jungfrauen op. 1 gemäß dem Vorbild von Schumanns Abegg-Variationen op. 1 komponiert. Der Komponist entdeckte das Vorgehen, ein „Thema“ auf die Buchstaben des Namens jenes Fräuleins auszuführen, welchem das Werk gewidmet ist. Zu Beginn der Komposition, unmittelbar nach dem Titel, werden aus dem Namen Sofija Sekulić drei Töne ausgeführt, welche der Komponist als Thema vorgibt: Sofia Sekulić (f-a-e). Nach der Exposition des Themas in der Einführung greift der Komponist es variiert in drei Walzernummern auf.
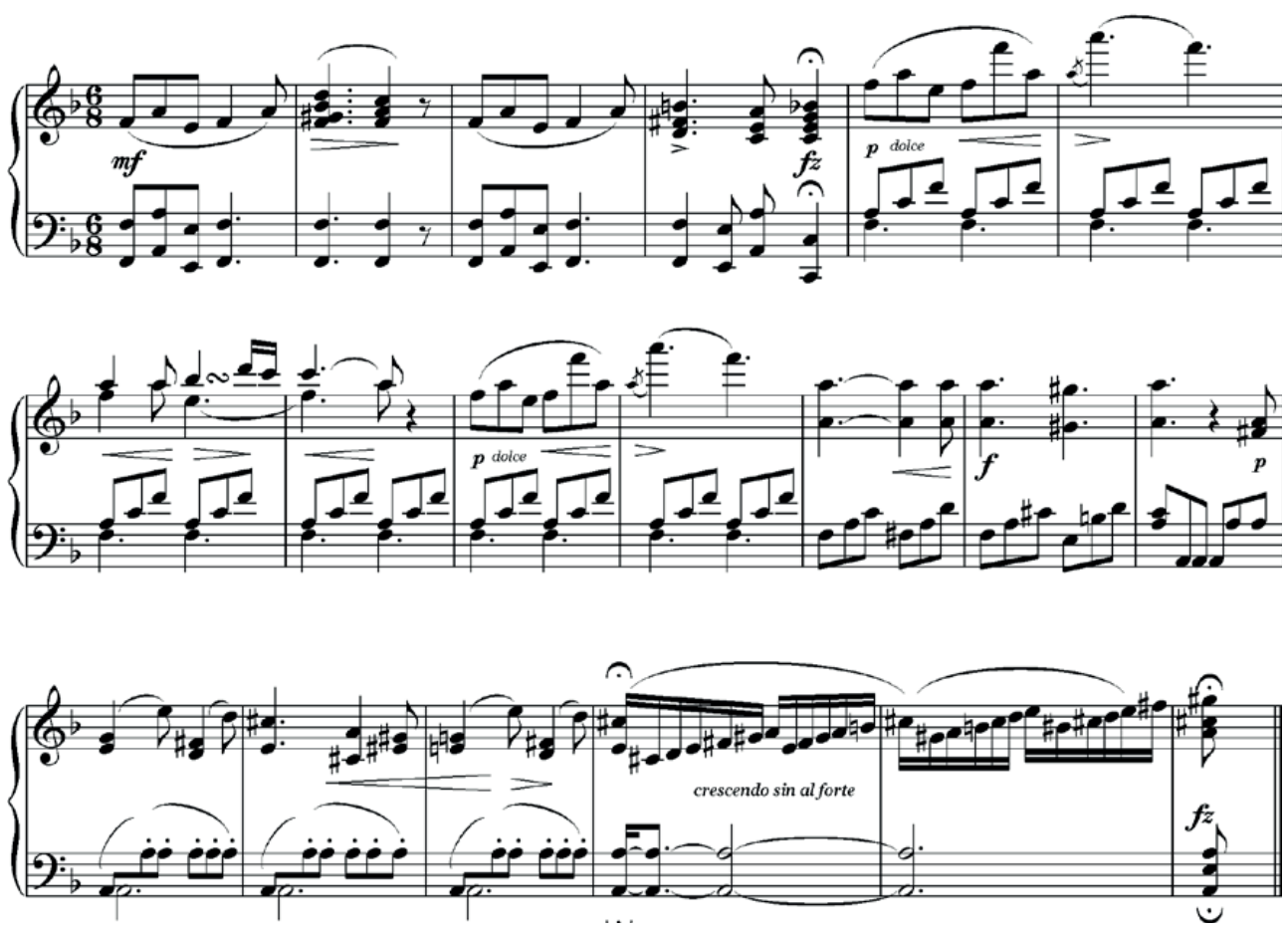

Notenbeispiel 1: Aleksandar Morfidis Nisis, Gruß an die serbischen Jungfrauen, op.1, T. 1-19.

Die Stilisierung internationaler Salontänze (Polka, Walzer, Quadrille, Polonaise, Cotillon) sind auch Nisis' erfolgreichsten Werken zu eigen. ${ }^{21}$ Aus den erhaltenen Konzertprogrammen sowie aus den Abschriften kann man Rückschlüsse auf seine Bearbeitungen serbischer Volkslieder ziehen, die in der Handschriftensammlung Chants Serbes beinhaltet sind. ${ }^{22}$

21 Das Kompositionsjahr der hier erwähnten Werke für Klavier von Morfidis Nisis ist nicht bekannt. Abgesehen vom Walzer Gruß an die serbischen Jungfrauen op.1 (1841) und dem Jelacic Marsch (1849) sind die weiteren Kompositionen von Nisis - dem bisherigen Erkenntnisstand nach - nicht gedruckt worden. Die Abschriften von Nisis' Kompositionen für Klavier, die aus dem Jahr 1870 stammen, sind im Album von Marija Panajotović in der Bibliothek der Matica Srpska in Novi Sad erhalten geblieben (Signatur: BMS MR I 8).

22 Das Entstehungsjahr der Sammlung ist nicht bekannt. In der Bibliothek der Matica Srpska wird die Abschrift dieser Sammlung aus dem Jahr 1870 aufbewahrt (BMS MR I 8 /Pv 19). 
Nach seiner Ankunft in Belgrad machte sich Alois Kalauz zunächst vor allem als Pianist und privater Klavierlehrer einen Namen. Bald jedoch begann er auch Salonmusik für Klavier zu komponieren, wobei er meistens Melodien serbischer Volkslieder und bürgerliche Lieder bearbeitete. Es handelt sich hier um internationale Salontänze (Polka, Quadrille, Marsch) sowie Variationen und Phantasien. Bald begann er auch systematisch, Melodien von Volksliedern aufzuzeichnen und zu sammeln. Die erste Sammlung von Volksliedern und -tänzen für Klavier veröffentlichte Kalauz in Wien unter dem Titel Serbische Melodien (1850, 1851). ${ }^{23}$ In dieser Sammlung sind insgesamt 43 Melodien serbischer Volkslieder enthalten. Beide Bände enthalten Vorworte sowohl in serbischer und französischer als auch in deutscher Sprache, in welchen der Autor klar sein Kriterium zur Sammlung der Lieder darlegt. Sodann geht er auf die Unterschiede zwischen Liedern des ländlichen und des städtischen Umfelds ein und unterscheidet zudem Lieder, in welchen fremde Einflüsse vorhanden sind, von authentischen Volksliedern. Er legt auch Augenmerk auf die tonale und rhythmische Eigenheit jener Lieder.

Das Wirken von Alois Kalauz entfaltete sich beginnend mit den 1850er Jahren parallel zur künstlerischen Tätigkeit von Kornelije Stanković, der in der serbischen Musikgeschichte als Begründer der nationalen Richtung in der Musik gilt. In Stankovićs Schaffen als Komponist, Pianist, Melograph und Dirigent sublimieren sich die Bestrebungen seiner Vorgänger, geben aber auch Leitlinien für die weitere Richtung im serbischen Musikschaffen des 19. Jahrhunderts vor. Von besonderer Bedeutung für seine künstlerische Vorliebe und sein Reifen war seine Ausbildung in Wien. Das reiche kulturelle und politische Leben der Stadt hinterließ bei dem jungen Künstler tiefe Spuren: einerseits die Kontakte mit dem serbischen und slowenischen Kreis und deren Ideen und andererseits besonders das Studium bei Simon Sechter, dem bedeutenden Komponisten, Pianisten, Musiktheoretiker und Professor am Konservatorium. In Wien entstanden die ersten Musikwerke Stankovićs: Walzer für Klavier und Lieder auf Verse von Goethe und Schiller. ${ }^{24}$ Es ist symbolträchtig, dass er zunächst auf Gedichte führender deutscher Dichter komponierte, und es ist bekannt, dass diese - insbesondere Goethe - Bewunderer und Propagatoren der serbischen Volksdichtung waren. ${ }^{25}$ Dank der Tätigkeit von Vuk Stefanović Karađić (1787-1864) ${ }^{26}$ sowie den direkten Ratschlägen des russisch-orthodoxen Erzpriesters Michail Fjodorowitsch Rajewski (1811-1884) und anderen slawischen Intellektuellen widmete sich Stanković dem Sammeln und Niederschreiben von serbischen geistlichen und weltlichen Volksliedern. ${ }^{27}$ Für Klavier komponierte er virtuose Salon-Variationen, internationale Salontänze (Walzer,

23 Serbische Melodien. Sammlung von National-Liedern und Tänzen: gesammelt für das Pianoforte gesetzt und der durchlauchtigsten Prinzesin Cleopatra Karadjordjewitsch Tochter des regierenden Fürsten Alexander von Serbien mit ehrerbietigster Hochachtung gewidmet, Heft I (1850); Heft II (1852), Wien.

24 Rudolf Flotzinger, „Der Musikunterricht des Kornelije Stanković in Wien um 1850“, in: Kornelije Stanković i njegovo doba [Kornelije Stanković und seine Zeit], hrsg. von Dimitrije Stefanović, Belgrad 1985, S. $42 \mathrm{f}$.

25 Die genannten Lieder von Kornelije Stanković gelten als verloren.

26 Er war Philologe und Reformer der serbischen Sprache, Sammler von volkstümlichen Kulturerbe und Autor des ersten Wörterbuches der serbischen Sprache (Wien, 1818).

27 Volkslieder bearbeitete er gewöhnlich für Stimme und Klavier, für Solo-Klavier oder für vierstimmigen Chor. 
Quadrille, Polka) sowie auch einfache Bearbeitungen serbischer Volkslieder und -tänze. Alle seine Kompositionen für Klavier wurden zwischen 1853 und 1863 in Wien veröffentlicht. ${ }^{28}$

\section{jahr}

1853

1854

1855

1857

1857

1862

1862
Werktitel

Variationen Serbe, steh auf, steh auf., op. 3

Variation Erinnerst du dich an jene Zeit, op. 4

Slawische Quadrille

Variationen Warum

kämpfen meine

Gedanken, op. 6
Herausgeber

Pietro Mechetti véuve, Vienna

Pietro Mechetti véuve, Vienna

\section{Widmung}

dem Prinzen Mihail M.

Obrenović

der Fürstin Julija M.

Obrenović, geborene

Gräfin Hunjadi

Pietro Mechetti sel. Witwe, dem Fräulein adelige Wien Helene Riđički von Skribešić

Gustav Albrecht, Wien

Der serbischen

Pancsovaer Gemeinde

Herrn Stephan M.

Georgievits

dem montenegrinischen

Fürsten Danilo I
den Serbinnen
A. Grube, Vienna

Gustav Albrecht, Wien

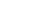

(1)

Serbische Volkslieder, gesetzt für Gesang und Klavier

Serbische Volkslieder, gesetzt für Gesang und

$$
\text { Serbische Quadrille }
$$

A. Grube, Vienna

Bulgarische Quadrille

Gustav Albrecht, Wien

den jungen Bulgarinnen

Brüderlichkeits-Polka

Gustav Albrecht, Wien

den Bulgaren

Serbische Volkslieder

Gustav Albrecht, Wien

dem serbischen Fürsten

Mihail Obrenović III

Erstes Buch

Serbische Volkslieder

für Gesang und Klavier,

Lith. G. Wegelein, Wien

dem Fürsten V.

P. Balabin, dem

kaiserlichen russischen

Gesandten in Wien

Tabelle 1: Wiener Ausgaben der Kompositionen für Klavier sowie für Stimme und Klavier von K. Stanković

28 Stanković veröffentlichte in Wien auch drei Bücher über traditionellen serbischen Kirchengesang $(1862,1863,1864)$. 


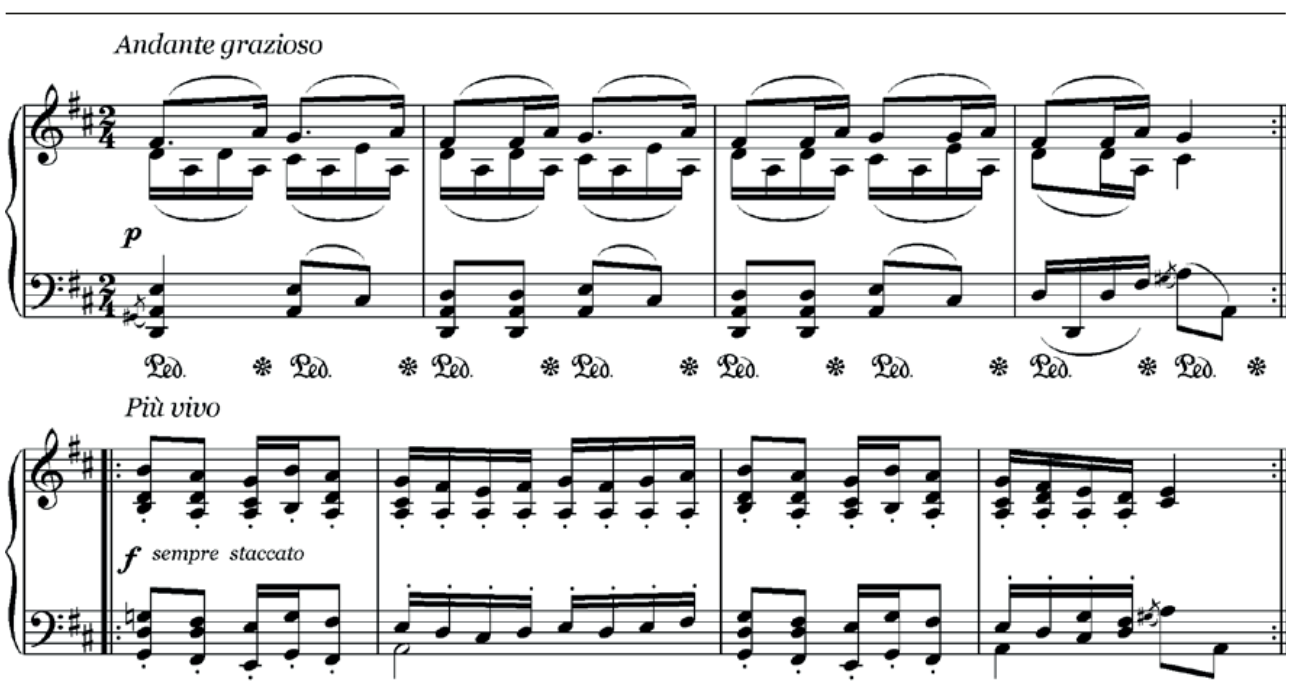

Notenbeispiel 2: Kornelije Stanković, Smederevka ${ }^{29}$

Als Pianist hatte Stanković Konzertauftritte in Wien, Budapest sowie auch in vielen anderen Städten in der Vojvodina und in Serbien. Neben eigenen Kompositionen spielte er auch Werke von Franz Liszt und Sigismund Thalberg, aber auch Werke der damals berühmten aber heute in Vergessenheit geratenen Salon-Pianisten und -Komponisten: Jacob Blumenthal (1829-1908), Louis Lacomb (1818-1884), Rudolf Willmers (1821-1878), Josef Eduard Maximilian Pirkhert (1817-1881).

Nach dem Tod von Stanković bedienten sich Musiker seiner Aufzeichnungen von Volksmelodien und veröffentlichten sie in Sammlungen jugoslawischer Gesänge. ${ }^{30}$ Aber auch heimische und fremde Komponisten bauten sie in ihre Werke ein. Dank der Aufzeichnungen von Kornelije Stanković können serbische Volksmelodien in Werken von Johann Strauß, Tschaikowski, Rimski-Korsakow und einer Reihe anderer Komponisten gefunden werden. ${ }^{31}$ In der Zeit der glanzvollen Entwicklung des Pianistentums und der Literatur für Klavier in Europa legte Kornelije Stanković einen Meilenstein im Schaffen von serbischer Klaviermusik. Beinahe alle seine Klavierkompositionen, die ausgesprochenen Saloncharakter aufweisen, basieren auf Melodien von Volksliedern und -tänzen. Sie reflektieren somit die Nachfrage des damaligen jungen serbischen Bürgertums und zeugen vom Gedankengut der nationalen Bewegung in der Kunst. Deshalb wurden sie mit wahrer Begeisterung aufgenommen, insbesondere bei der serbischen Jugend.

29 Smederevka wurde innerhalb der Sammlung Serbische Volkslieder 1863 in Wien veröffentlicht.

30 Zum Beispiel Südslawische Volkslieder, hrsg. Franjo Kuhač, Band, I -V, Zagreb 1878, 1879, 1880, 1881, 1941.

31 Johann Strauss, Slaven Potpourri op. 39 (nach K. Stanković: Sirmir kolo); P. I. Tschaikowski, Slawischer Marsch b-Moll op. 31 (nach K. Stanković: Helle Sonne, du scheinst nicht gleich; Dies ist die Schwelle des geliebten Serbentums und Gerne geht der Serbe zu den Soldaten); N. Rimsky-Korsakow, Fantasie über serbische Themen op. 6 (nach K. Stanković: Helle Sonne, du scheinst nicht gleich; Du Mädchen) usw. Đorđe Perić, „Bibliografija Kornelija Stankovića“, in: Kornelije Stanković i njegovo doba [Kornelije Stanković und seine Zeit], hrsg. von Dimitrije Stefanović, Belgrad 1985, S. 287-326. 


\section{Schlussfolgerung}

Nach dem Tod von Stanković folgte die neue Komponistengeneration in ihrem Schaffen seinem Modell. Bis zum Ende des Ersten Weltkriegs waren parallel mit ihnen auch zahlreiche tschechische Komponisten aktiv. Hinsichtlich der Entwicklung des Musiklebens haben auch Gastauftritte von Künstlern einen bedeutenden Beitrag geleistet, ebenso wie die Entwicklung des Musikalienhandels. Über die weitverzweigten Kanäle des Musikmarktes - sowohl des reproduzierenden als auch des verlegenden - hat sich ein internationales Repertoire verbreitet. Im Angebot der heimischen Musikverleger nahm bis zu Beginn des 20. Jahrhunderts die Salonmusik für Klavier einen bedeutenden Platz ein (internationale Salontänze, Variationen, Phantasien und Potpourris, Bearbeitungen serbischer Volkslieder und Volkstänze, Märsche). Das Publikum nahm die heraushörbaren Volksmelodien wie auch die Melodien von Liedern auf die Vaterlandsliebe - genannt „budnica“ (dt. Aufwecker) - mit unverhohlener Begeisterung auf. Es handelte sich im Falle der Volkslieder und -tänze um verborgene patriotische Signale oder, um sich der Worte Schumanns für Chopins Polonaisen zu bedienen, um ,,von Blumen verdeckte Kanonen“. Es scheint, als würde gerade der Inhalt bzw. die Botschaft des musikalischen Werkes den Wert der Komposition ausmachen: Das Hervorheben nationaler Charakteristika mittels Musik dominierte im 19. Jahrhundert.

Von besonderer Bedeutung für die weitere Entwicklung der serbischen Musik und deren Eingliederung in die damaligen Strömungen der europäischen Musik waren die Errungenschaften in der Ausbildung der jungen Generation an europäischen Konservatorien, von wo sie sodann wieder mit neuen Kenntnissen und Erfahrungen in ihre Heimat zurückkehrten. Bereits ab den 1850er Jahren erhielten die Komponisten ihre Ausbildung vorwiegend in Prag (Aksentije Maksimović, Jovan Paču, Mita Topalović, Josif Marinković, Božidar Joksimović), Wien (Kornelije Stanković, Josif Marinković, Petar Krstić, Petar Stojanović), Leipzig (Stevan Stojanović Mokranjac) und München (Stevan Stojanović Mokranjac, Stanislav Binički), und nach dem Ersten Weltkrieg auch in Frankreich (Stanislav Binički, Milenko Paunović, Miloje Milojević, Vladimir Đorđević) und in England (Kosta Manojlović). In den letzten Jahrzehnten des 19. Jahrhunderts trat eine Ablehnung von Salonkultur zutage, sowohl im Konzertrepertoire als auch im kompositorischen Schaffen der jungen Künstler, welche hervorragende Absolventen von europäischen Konservatorien waren. Dieser Wandel wird auch in Presseartikeln offenkundig, wo man ab den 1880er Jahren auf kritische Anmerkungen über Salonmusik stößt, worüber ,,spekulative Herausgeber lächeln und die Musen weinen". 32 Indessen haben sich in der ersten Hälfte des 19. Jahrhunderts ausgerechnet im Rahmen von Hausmusik und Salonmusik dank des Wirkens fremder sowie einheimischer Musiker und Amateure die Grundlagen für die Entwicklung von Musikeinrichtungen, Musikunterricht an Schulen und das Konzertleben herausgebildet. Da es im öffentlichen Leben nicht viele Gelegenheiten gab, Musik zu hören, stellte das Musizieren im Salon eine Ergänzung und in kleineren Orten ein Ersatz zu öffentlichen Konzerten dar. Der private Salon in seinen unterschiedlichen Erscheinungsformen ersetzte das Fehlen von öffentlichen Vereinigungen und professionellen Einrichtungen, die sich zu jener Zeit erst herauszubilden begannen. Die Voraussetzungen für deren Entwicklung und Existenz wurden nach und nach geschaffen: das Bewusstsein für deren Notwendigkeit, organisatorische und technische Voraussetzungen, Mittel zu ihrer Realisierung und vor allem politische und rechtliche Möglichkeiten.

32 Robert Tolinger, „Nekoliko reči o kućevnoj nezi glazbe“ [Einige Worte über die häusliche Musikpflege], in: Gudalo 7 (1886), S. 131. 